\title{
AUTOMORPHISMS OF NONSELFADJOINT DIRECTED GRAPH OPERATOR ALGEBRAS
}

\author{
BENTON L. DUNCAN
}

(Received 25 May 2007; accepted 28 August 2008)

Communicated by G. A. Willis

\begin{abstract}
We analyze the automorphism group for the norm closed quiver algebras $\mathcal{T}^{+}(Q)$. We begin by focusing on two normal subgroups of the automorphism group which are characterized by their actions on the maximal ideal space of $\mathcal{T}^{+}(Q)$. To further discuss arbitrary automorphisms we factor automorphism through subalgebras for which the automorphism group can be better understood. This allows us to classify a large number of noninner automorphisms. We suggest a candidate for the group of inner automorphisms.
\end{abstract}

2000 Mathematics subject classification: primary 47L40; secondary 46L80, 47L55, 47L75.

Keywords and phrases: graph operator algebras, automorphisms.

Building on work of Davidson and Pitts [3], we analyze the automorphism group of the norm closed quiver algebras $\mathcal{T}^{+}(Q)$. We use the maximal ideal space and the underlying directed graph to make a first pass at the automorphism group of $\mathcal{T}^{+}(Q)$. After this we use a finer analysis and graph subalgebras to describe large classes of automorphisms.

One thing that comes out of this paper is a clearer understanding of when questions concerning continuity of automorphisms arise for the quiver algebras. In particular, if the graph has a source and a sink and an infinite number of cycleless paths between a source and a sink then continuity of automorphisms is not guaranteed.

We begin by describing two normal subgroups of $\operatorname{Aut}\left(\mathcal{T}^{+}(Q)\right)$ : the component fixing automorphisms, $\mathrm{CF}(Q)$, and the Gelfand fixing automorphisms, $\mathrm{GF}(Q)$. Both $\mathrm{CF}(Q)$ and $\mathrm{GF}(Q)$ are related to how the automorphism acts on the maximal ideal space of $\mathcal{T}^{+}(Q)$. The quotient of $\operatorname{Aut}\left(\mathcal{T}^{+}(Q)\right)$ by the component fixing automorphisms $\mathrm{CF}(Q)$ is in one-to-one correspondence with the $\operatorname{group} \operatorname{v-Aut}(Q)$, a

(C) 2009 Australian Mathematical Publishing Association Inc. 1446-7887/2009 \$16.00 
subgroup of the automorphism group of the graph. Lastly the natural quotient yields a split exact sequence of groups.

The subgroup of Gelfand fixing automorphisms is more complicated. Here the natural quotient does not form a split exact sequence. However, the quotient, as in [3], is a direct sum of copies of $\operatorname{Aut}\left(\mathbb{B}_{n}\right)$ the conformal automorphisms of the unit ball of $\mathbb{C}^{n}$.

We also see that every Gelfand fixing automorphism factors through graph subalgebras arising from consecutive vertices. We then analyze such subgraphs and their automorphism groups completely. A further refinement is then made to look at those automorphisms which factor naturally through subalgebras arising from loopless cycles. This analysis relies on the paper of Alaimia [1]. We are then left with a normal $\operatorname{subgroup}, \operatorname{MIF}(Q)$, which we conjecture is equal to $\operatorname{Inn}\left(\mathcal{T}^{+}(Q)\right)$.

The weak operator topology closed free semigroupoid algebras are slightly more complicated in the sense that continuity of automorphisms is less tractable. However, proper modifications of the results in this paper, in particular the techniques, will apply in most cases to the free semigroupoid algebras.

\section{Directed graphs and their algebras}

We begin with definitions and terminology. We can view a directed graph $Q$ as a four-tuple $(V(Q), E(Q), r, s)$ given by a pair of sets, $V(Q)$ and $E(Q)$, and a pair of maps $r: E(Q) \rightarrow V(Q)$ and $s: E(Q) \rightarrow V(Q)$. We assume, of course, that $V(Q)$ is nonempty and where it will not cause confusion we often write $V$ for $V(Q)$ and $E$ for $E(Q)$. We call the set $V$ the vertices of $Q$ and the set $E$ the edges of $E$. The maps $r$ and $s$ are called the range map and source map, respectively. Given a pair of vertices $v, w$ denote by $E_{v, w}$ the edges $e$ with $s(e)=w$ and $r(e)=v$.

In this paper we assume that both $V$ and $E$ are countable. A vertex $v$ is a source if $r(e) \neq v$ for all $e \in E$ and we say a vertex $v$ is a sink if $s(e) \neq v$ for all $e \in E$. We say that the graph $Q$ has no sources if the range map is onto and we say that $Q$ has no sinks if the source map is onto. We say that $Q$ is infinite in one direction if either the range map or the source map is onto.

A finite path $w$ in a directed graph is a finite sequence of edges $w=e_{1} e_{2} \cdots e_{n}$ such that $r\left(e_{i}\right)=s\left(e_{i-1}\right)$ for all $i \geq 2$. The length of the finite path $w$ will be the number of edges in the sequence; we denote this number by $l(w)$. A vertex can be considered a path of length zero; we call these degenerate paths when used in this way. We say that a path $w=e_{1} e_{2} \cdots e_{n}$ is a cycle if $s\left(e_{n}\right)=r\left(e_{1}\right)$. We call a cycle nontrivial if the length of the cycle is greater than one. If the length of a cycle is one we call the cycle a loop. We say that a vertex $v$ supports the path $w$ if there is $e_{i}$ in the path $w$ such that $s\left(e_{i}\right)=v$.

Given a directed graph $Q$ we let $\ell^{2}(Q)$ denote the Hilbert space of sequences indexed by the finite directed paths in $Q$. Here we are including the degenerate paths. Now consider the left regular representation of $Q$ acting on $\ell^{2}(Q)$ by concatenation of paths. The vertices $v \in V(Q)$ give rise to a family of orthogonal projection $P_{v}$ 
which sum to the identity. Every edge $e$ gives rise to a partial isometry $L_{e}$ such that $L_{e}^{*} L_{e}=P_{s(e)}$ and $\sum_{e: r(e)=v} L_{e} L_{e}^{*}=P_{r(e)}$. The norm closed nonselfadjoint algebra generated by $\left\{L_{e}, P_{v}: e \in E, v \in V\right\}$ will be denoted $\mathcal{T}^{+}(Q)$. Although it is not addressed in this paper one can also look at the weak operator topology closure of this algebra, denoted $\mathcal{L}_{Q}$. This latter algebra is called the free semigroupoid algebra associated to $Q$. We refer the reader to [6] and [7] for more information about these algebras.

An important aspect of these algebras is that given $X \in \mathcal{T}^{+}(Q)$ there is a unique 'Fourier series' associated to $X$. In particular,

$$
X=\sum_{w} a_{w} L_{w}
$$

where $a_{w}$ is a complex scalar and $w$ varies over all possible finite paths in $Q$. Of course, the Fourier series of a product is the usual convolution product of the two Fourier series. In addition, given any $n \geq 0$ there is an ideal

$$
\mathcal{T}^{+}(Q)_{n}=:\left\{X=\sum a_{w} L_{w} \mid a_{w}=0 \text { for all } w \text { with } l(w)<n\right\} .
$$

In the case of $n=1$ this ideal is generated by $\left\{L_{e} \mid e \in E\right\}$.

Lastly, given a Banach algebra $A$ we denote by $\operatorname{Aut}(A)$ the set of continuous automorphisms of $A$. We use $\operatorname{Inn}(A)$ to denote the set of those automorphisms on $A$ given by $X \mapsto z X z^{-1}$ where $z$ is invertible in $A$, the so-called inner automorphisms.

\section{Directed graph automorphisms}

We begin by analyzing directed graph automorphisms with an eye to using these to study the automorphisms of $\mathcal{T}^{+}(Q)$. If $Q_{1}$ and $Q_{2}$ are directed graphs then by a directed graph homomorphism $\theta: Q_{1} \rightarrow Q_{2}$ we mean a pair of maps $\theta_{V}: V\left(Q_{1}\right) \rightarrow$ $V\left(Q_{2}\right)$ and $\theta_{E}: E\left(Q_{1}\right) \rightarrow E\left(Q_{2}\right)$ such that the following diagrams commute.
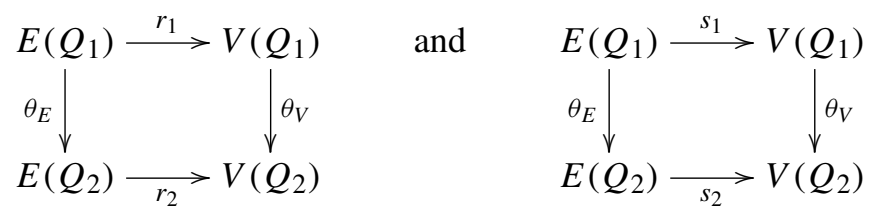

In other words $s_{2}\left(\theta_{E}(e)\right)=\theta_{V}\left(s_{1}(e)\right)$ and $r_{2}\left(\theta_{E}(e)\right)=\theta_{V}\left(r_{1}(e)\right)$ for all edges $e \in E\left(Q_{1}\right)$. Here $r_{i}$ and $s_{i}$ are the range and source maps on the respective graphs. We refer to $\theta_{V}$ as a vertex map and $\theta_{E}$ as an edge map. As expected we say that $\theta$ is a directed graph isomorphism if both $\theta_{V}$ and $\theta_{E}$ are one-to-one and onto. If $\theta: Q \rightarrow Q$ is an isomorphism we call it a directed graph automorphism. We find it useful to study the directed graph automorphisms in our analysis of the automorphisms of quiver algebras. 
We begin with some elementary lemmas for which the reader can readily provide proofs. We start by noting that the map id: $Q \rightarrow Q$ given by $\operatorname{id}_{V}(v)=v$ and $\operatorname{id}_{E}(e)=e$ is an automorphism. Further, given two automorphisms $\theta_{1}$ and $\theta_{2}$ we write $\theta_{1} \circ \theta_{2}$ to denote the composition of the respective vertex and edge maps.

LEMMA 2.1. If $\theta$ is an automorphism of $Q$, then there exists an automorphism $\theta^{-1}$ such that

$$
\theta \circ \theta^{-1}=\theta^{-1} \circ \theta=\mathrm{id} .
$$

Note that to define $\theta^{-1}$ we need only look at the reverse set maps $\theta_{V}^{-1}$ and $\theta_{E}^{-1}$ which are well defined because $\theta_{V}$ and $\theta_{E}$ are both one-to-one and onto. The proof consists of verifying that these new maps give rise to the appropriate commutative diagrams. The next lemma is now trivial.

LEMMA 2.2. The set of automorphisms of a directed graph, call it $\operatorname{Aut}(Q)$, is a group under composition, with identity element id.

Definition 2.3. Let $\operatorname{VF}(Q)$ denote the set $\left\{\theta \in \operatorname{Aut}(Q) \mid \theta_{V}=\operatorname{id}_{V}\right\}$. We call this set the vertex fixing automorphisms of $Q$.

PROPOSITION 2.4. The set $\operatorname{VF}(Q)$ is a normal subgroup of $\operatorname{Aut}(Q)$.

Proof. Note that $\operatorname{VF}(Q)$ is closed under composition, $\operatorname{id} \in \operatorname{VF}(Q)$, and if $\theta_{V}=\mathrm{id}_{v}$, then $\theta_{V}^{-1}=\mathrm{id}_{V}$ and, hence, $\operatorname{VF}(Q)$ is a subgroup of $\operatorname{Aut}(Q)$. Now note that if $\theta$ is an arbitrary element of $\operatorname{Aut}(Q)$ then

$$
\theta_{V} \circ \mathrm{id}_{V} \circ \theta_{V}^{-1}=\theta_{V} \circ \theta_{V}^{-1}=\mathrm{id}_{V}
$$

and, hence, $\operatorname{VF}(Q)$ is normal inside $\operatorname{Aut}(Q)$.

Definition 2.5. Let v-Aut $(Q)$ denote the quotient $\operatorname{Aut}(Q) / \operatorname{VF}(Q)$ and call this group the vertex automorphisms of $Q$.

For concreteness we now present some examples.

EXAMPLE. Let $Q$ be the graph

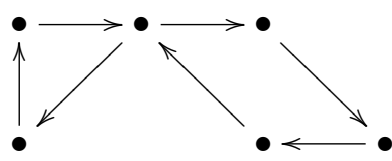

and note that the automorphism group is the trivial group.

EXAMPLE. Let $Q$ be the graph

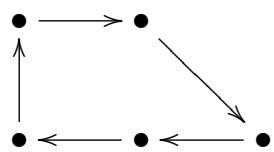

and note that while $\operatorname{Aut}(Q) \cong \mathbb{Z}_{5}$ the subgroup $\operatorname{VF}(Q)$ is trivial. 
EXAMPLE. As a final example let $Q$ be the graph

and note that $\operatorname{Aut}(Q) \cong \operatorname{VF}(Q) \cong S_{3}$.

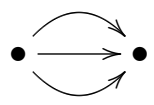

For the rest of the paper we only need v-Aut $(Q)$ but for the interested reader we complete our description of $\operatorname{Aut}(Q)$ for a countable directed graph $Q$. Let $E_{v, w}$ denote the set of edges in $Q$ such that $r(e)=v$ and $s(e)=w$, note that we do not require that $v \neq w$. Now assign to each $E_{v, w}$ a total ordering. In other words if there are $n$ edges in $E_{v, w}$ label them as $e_{1}, e_{2}, \ldots, e_{n}$. Note that an automorphism will map $E_{v, w}$ to $E_{v^{\prime}, w^{\prime}}$ for some vertices $v^{\prime}$ and $w^{\prime}$ and note that the cardinality of $E_{v, w}$ will be equal to the cardinality of $E_{v^{\prime}, w^{\prime}}$. We say that an automorphism $\theta$ preserves order at $(v, w)$ if $\theta_{E}\left(e_{i}\right)=f_{i}$, for all $e_{i} \in E_{v, w}$. Let $\operatorname{OP}(Q)$ denote the set automorphisms which preserve order at $(v, w)$ for all pairs $(v, w) \in V \times V$.

Proposition 2.6. The group v-Aut $(Q)$ is isomorphic to $\mathrm{OP}(Q)$.

Proof. Let $\theta \in \operatorname{Aut}(Q)$ and define $\theta^{\prime} \in \mathrm{OP}(Q)$ by $\theta_{V}^{\prime}(v)=\theta_{V}(v)$ for all vertices $v$. Furthermore, for $e_{i} \in E_{v, w}$, let $\theta_{E}^{\prime}\left(e_{i}\right)=f_{i}$, where $f_{i} \in E_{\theta_{V}}(v), \theta_{V}(w)$, then it is clear that $\theta^{\prime}$ is an automorphism and $\theta^{\prime} \in \mathrm{OP}(Q)$.

Now for $[\theta] \in \mathrm{v}-\operatorname{Aut}(Q)$ define the map $\Lambda \mid \mathrm{v}-\operatorname{Aut}(Q) \rightarrow \mathrm{OP}(Q)$ by $\Lambda([\theta])=\theta^{\prime}$. We first verify that this map is well defined. Let $\theta_{1} \circ \theta_{2}^{-1}=\sigma \in \operatorname{VF}(Q)$. Then note that $\theta_{1} \circ \theta_{2}^{-1}(v)=v$. It follows that $\left(\theta_{1}\right)_{V}=\left(\theta_{2}\right)_{V}$ and, hence, $\theta_{1}^{\prime}=\theta_{2}^{\prime}$ and $\Lambda$ is well defined.

Next note that $\Lambda([\mathrm{id}])=\mathrm{id}^{\prime}=\mathrm{id}$ and, finally,

$$
\Lambda([\theta] \circ[\sigma])=\Lambda([\theta \circ \sigma])=(\theta \circ \sigma)^{\prime} .
$$

Now $(\theta \circ \sigma)_{V}=\theta_{V} \circ \sigma_{V}$ and, hence, $(\theta \circ \sigma)^{\prime}=\theta^{\prime} \circ \sigma^{\prime}$ and the map $\Lambda$ is a homomorphism.

Now $\theta^{\prime} \in \operatorname{Aut}(Q)$ and $\Lambda\left[\theta^{\prime}\right]=\theta^{\prime}$ making $\Lambda$ onto. We need only verify that $\tau$ is one-to-one. So let $\Lambda([\theta])=\Lambda([\sigma])$. Then, in particular, $\theta_{V}=\sigma_{V}$ so $\theta_{V} \circ \sigma_{V}^{-1}=\mathrm{id}$. It follows that $[\theta]=[\sigma]$ and the result follows.

If we look at the map $\Lambda$ we note that in $\operatorname{v-Aut}(Q),[\theta]=\left[\theta^{\prime}\right]$ and hence the group $\operatorname{Aut}(Q)$ splits as

$$
\mathrm{VF}(Q) \oplus \mathrm{v}-\operatorname{Aut}(Q) \text {. }
$$

We can also analyze the group $\operatorname{VF}(Q)$ to obtain more information about $\operatorname{Aut}(Q)$. Recall that the symmetric group $S_{n}$ is the group of permutations on an $n$-element set.

PROpOSITION 2.7. The group $\operatorname{VF}(Q)$ is isomorphic to

$$
\bigoplus_{(v, w) \in V \times V} S_{n_{v, w}},
$$

where $n_{v, w}$ is the number of edges e with $r(e)=v, s(e)=w$. 
PRoof. If $\theta_{V}(v)=v$, then $\theta_{E}\left(E_{v, w}\right)$ is just a permutation of $E_{v, w}$. If we let $P_{v, w}$ denote the automorphisms which are the identity on $V$ and on $E_{v^{\prime}, w^{\prime}}$ where $v^{\prime} \neq v$ and $w^{\prime} \neq w$. Then $P_{v, w}$ is a normal subgroup of $\operatorname{VF}(Q)$. Further, if $v^{\prime} \neq v$ and $w^{\prime} \neq w$, then $P_{v, w} \cap P_{v^{\prime}, w^{\prime}}=\{$ id $\}$. Lastly, if $\theta \in \operatorname{VF}(Q)$, then for $(v, w) \in V \times V$ let $\theta_{v, w}$ be that element of $\operatorname{VF}(Q)$ such that $\theta_{v, w}(e)=\theta(e)$ for all $e \in E_{v, w}$ and $\theta_{v, w}(e)=e$ for all $e \notin E_{v, w}$. Then note that $\theta=\prod_{(v, w) \in v \times V} \theta_{v, w}$. In other words,

$$
\operatorname{VF}(Q)=\prod_{(v, w) \in v \times V} P_{v, w},
$$

and hence $\operatorname{VF}(Q)$ is the direct product of the $P_{v, w}$. It is easy to see that $P_{v, w}=S_{n_{v, w}}$ and the result follows.

Summarizing the above we have the following theorem.

THEOREM 2.8. Let $Q$ be a directed graph, then

$$
\operatorname{Aut}(Q) \cong \operatorname{v-Aut}(Q) \oplus\left(\bigoplus_{(v, w) \in V \times V} S_{n_{v, w}}\right)
$$

where $n_{v, w}$ is the number of edges e with $r(e)=v, s(e)=w$.

The importance of graph automorphisms is encoded in the following easy proposition.

Proposition 2.9. Let $\theta: Q \rightarrow Q$ be a graph automorphism, then there exists $a$ continuous automorphism $\tilde{\theta}: \mathcal{T}^{+}(Q) \rightarrow \mathcal{T}^{+}(Q)$.

PROOF. This is actually just a corollary of [2, Corollary 3.2] by noting that the left regular representation of a directed graph is pure and that $\theta$ induces a relabeling of $Q$ with respect to the left regular representation.

There are, of course, many automorphisms of $\mathcal{T}^{+}(Q)$ which do not come from $\operatorname{Aut}(Q)$. We look at these in the following.

\section{The maximal ideal space of a quiver algebra}

An important invariant for the algebras $\mathcal{T}^{+}(Q)$ is the space of multiplicative linear functionals, the maximal ideal space $M_{Q}$. This was studied in [5] in analyzing isomorphisms of directed graph operator algebras. We go through the description here and work through the properties that are necessary for what follows.

Let $v$ be a vertex in $Q$, then the projection associated with $v$, call it $P_{v}$, is orthogonal to the projections $P_{w}$ associated to different vertices $w$. As $P_{v}$ is a projection, the image of $P_{v}$ under a multiplicative linear functional must be equal to zero or one. Note that if it is equal to one, then by orthogonality, every other projection $P_{w}$ must be sent to zero. Hence, the first thing to know about the maximal ideal space is that $M_{Q}$ has a distinct component for each vertex in $Q$. 
Now fix $v \in V(Q)$ and let $e$ be an edge in $E(Q)$. Let $\pi$ be a multiplicative linear functional that sends $P_{v}$ to 1 . Then if $r(e) \neq v$ or $s(e) \neq v$, then $\pi\left(L_{e}\right)=0$. Let $E_{v, v}=\left\{e_{1}, e_{2}, \ldots, e_{n}\right\}$ and note that $\left|\left(\pi\left(L_{e_{1}}\right), \pi\left(L_{e_{2}}\right), \ldots, \pi\left(L_{e_{n}}\right)\right)\right| \leq 1$ since $\pi$ must be completely contractive. Similarly if $\lambda:=\left(\lambda_{1}, \lambda_{2}, \ldots, \lambda_{n}\right)$ is an element of $\mathbb{C}^{n}$ such that $|\lambda| \leq 1$, then the map which sends $P_{v}$ to 1 and $L_{e_{i}}$ to $\lambda_{i}$ defines a multiplicative linear functional on $\mathcal{T}^{+}(Q)$.

In fact, the following proposition is essentially Corollary 3.3 of [5] with the following notation. The nonnegative integer $n(v)$ denotes the number of loop edges supported by $v$ and $\mathbb{B}_{n(v)}$ denotes the unit ball in $\mathbb{C}^{n(v)}$, if $n(v)>0$ and $\{0\}$ if $n(v)=0$.

PROPOSITION 3.1 (Katsoulis-Kribs [5]). If $Q$ is a countable directed graph, then $M_{Q}$ is a locally compact Hausdorff space with a connected component corresponding

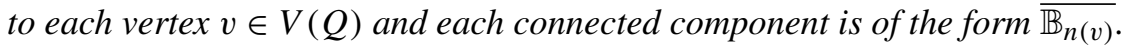

Implicit in this statement is that the $w^{*}$-topology on a connected component of $M_{Q}$ corresponds to the usual topology on $\mathbb{C}^{n(v)}$. Note that if $v$ is a sink or a source, then the component corresponding to $v$ is $\{0\}$. In fact, the component corresponding to $v$ is $\{0\}$ if and only if $v$ does not support a loop edge. The following is a strengthening of a standard fact for Banach algebras. In particular, if an automorphism of a Banach algebra is continuous, then the result follows immediately. We use the special structure of the algebra $\mathcal{T}^{+}(Q)$ to see that continuity is not necessary.

THEOREM 3.2. Let $\theta$ be a (not necessarily continuous) automorphism of $\mathcal{T}^{+}(Q)$, then $\theta$ induces a homeomorphism $M_{\theta}: M_{Q} \rightarrow M_{Q}$.

The proof begins with a few lemmas.

Lemma 3.3. Let $X \in \mathcal{T}^{+}(Q)_{1}$ such that $X^{2}=X$, then $X=0$.

Proof. Let $X \in \mathcal{T}^{+}(Q)_{1}$, then

$$
X=\sum_{l(w) \geq 1} a_{w} L_{w} .
$$

Let $m=\inf \left\{l(w) \mid a_{w} \neq 0\right\}$. Now looking at

$$
X^{2}=\sum_{l(w) \geq 1} b_{w} L_{w}
$$

we see that $n=\inf \left\{l(w) \mid b_{w} \neq 0\right\} \geq 2 m$. Thus, if $X^{2}=X$, then $m=\infty$ and $n=\infty$ and, hence, $a_{w}=0$ for all finite paths $w$.

LEMMA 3.4. Let $v$ be a vertex in $Q$ and $\theta$ a (not necessarily continuous) automorphism of $\mathcal{T}^{+}(Q)$. Then there exists a unique vertex $v^{\prime}$ such that $\theta\left(P_{v}\right)=P_{v^{\prime}}+X$ where $X \in \mathcal{T}^{+}(Q)_{1}$.

PRoOF. Note that $\left(P_{v}\right)^{2}=P_{v}$ and, hence, $\left(\theta\left(P_{v}\right)\right)^{2}=\theta\left(P_{v}\right)$. We know by the preceding lemma that $\theta\left(P_{v}\right) \notin \mathcal{T}^{+}(Q)_{1}$ and, hence, $\theta\left(P_{v}\right)=\sum_{w \in V} a_{w} P_{w}+X$, 
where $X \in \mathcal{T}^{+}(Q)_{1}$. We also know that $a_{w} \in\{0,1\}$ for all $w \in V$, otherwise $\left(\theta\left(P_{v}\right)\right)^{2} \neq \theta\left(P_{v}\right)$. Now, if $v_{1} \in V$ with $v_{1} \neq v$, then $P_{v} P_{v_{1}}=0=P_{v_{1}} P_{v}$ and, hence, if $a_{w}=1$, then writing $\theta\left(P_{v_{1}}\right)=\sum_{w \in V} b_{w} P_{w}+Y$ with $Y \in \mathcal{T}^{+}(Q)_{1}$ we know that $b_{w}=0$.

Now note that if $r(e) \neq s(e)$, then $L_{e}^{2}=0$ and, hence, $\theta\left(\left(L_{e}\right)\right)^{2}=0$. However, if $\theta\left(L_{e}\right) \notin \mathcal{T}^{+}(Q)_{1}$, then $\theta\left(L_{e}\right) \neq 0$ which is a contradiction. If $r(e)=s(e)=v$ the situation is more complicated, we have to allow for the possibility (which can certainly happen) that $\theta\left(L_{e}\right)=\sum_{w \in V} \alpha_{w} P_{w}+Z$ where $Z \in \mathcal{T}^{+}(Q)_{1}$. Writing $\theta\left(P_{r(e)}\right)=$ $\sum_{w \in V} a_{w} P_{w}+X_{e}$, then since $L_{e} P_{r(e)}=L_{e}=P_{r(e)} L_{e}$ we know that $\alpha_{w} \neq 0$ only if $a_{w} \neq 0$. Further since $L_{e}^{2}-L_{e}=L_{e}\left(L_{e}-P_{r(e)}\right)$ we have

$$
\begin{aligned}
\sum_{w \in V}\left(\alpha_{w}^{2}-\alpha_{w}\right) P_{w}+Z_{1} & =\theta\left(L_{e}^{2}-L_{e}\right) \\
& =\theta\left(L_{e}\right) \theta\left(L_{e}-P_{r(e)}\right) \\
& =\left(\sum_{w \in V} \alpha_{w} P_{w}+Z\right)\left(\sum_{w \in V}\left(\alpha_{w}-a_{w}\right) P_{w}+Z_{2}\right) \\
& =\sum_{w \in V}\left(\alpha_{w}^{2}-\alpha_{w} a_{w}\right) P_{w}+Z_{3} \\
& =\sum_{w \in V}\left(\alpha_{w}^{2}-a_{w}\right) P_{w}+Z_{3}
\end{aligned}
$$

where $Z_{1}, Z_{2}, Z_{3} \in \mathcal{T}^{+}(Q)_{1}$. Hence, if there is some $w$ with $\alpha_{w} \neq 0$, then $\left(\alpha_{w}^{2}-\alpha_{w}\right)=\left(\alpha_{w}^{2}-a_{w}\right)$ for all $w$ and, hence, $\alpha_{w}=a_{w}$ for all $w \in V$.

It follows that if there exist $v^{\prime}$ and $v^{\prime \prime}$ such that $a_{v^{\prime}}=a_{v^{\prime \prime}}$, then neither $P_{v^{\prime}}$ nor $P_{v^{\prime \prime}}$ are in the range of $\theta$ contradicting the fact that $\theta$ is an automorphism.

We say that a path $w=e_{1} e_{2} \cdots e_{n}$ in $Q$ is vertex acyclic if $r\left(e_{i}\right) \neq r\left(e_{j}\right)$ for all $i \neq j$. We say that a vertex $v$ is sinking if for every finite path $w$ with $s(w)=v$ we have that $w$ is vertex acyclic. Denote the set of sinking vertices by $V_{s}$. Let $Q_{0}$ denote the graph obtained from $Q$ be removing all vertices in $V_{s}$ and all edges with $r(e) \in V_{s}$. Let $Q_{s}$ denote the graph obtained by removing all vertices not in $V_{s}$ and all edges with $s(e) \notin V_{s}$. The following lemma follows trivially from the description of the maximal ideal space $M_{Q}$ as corresponding to vertices.

Lemma 3.5. Let $Q$ be a directed graph then $M_{Q_{0}}$ is homeomorphic to a locally compact Hausdorff subspace of $M_{Q}$. Here $M_{Q_{s}}$ is homeomorphic to a locally compact Hausdorff subspace of $M_{Q}$. The two subspaces are disjoint and under this identification $M_{Q}=M_{Q_{0}} \cup M_{Q_{s}}$.

We now come to the central lemma which yields the homeomorphism induced by $\theta$. 
LEMMA 3.6. Let $\theta$ be a (not necessarily continuous) automorphism of $\mathcal{T}^{+}(Q)$, then $\theta$ induces a bijective mapping $\widehat{\theta}: M_{Q} \rightarrow M_{Q}$ such that $\left.\widehat{\theta}\right|_{M_{Q_{0}}}$ is a bijective mapping onto $M_{Q_{0}}$ and $\left.\widehat{\theta}\right|_{Q_{S}}$ is a bijective mapping onto $M_{Q_{s}}$.

Proof. This follows since $\mathcal{T}^{+}(Q)$ can be written in lower triangular form as

$$
\left[\begin{array}{ll}
A & 0 \\
C & D
\end{array}\right]
$$

where $A \in \mathcal{T}^{+}\left(Q_{0}\right), D \in \mathcal{T}^{+}\left(Q_{s}\right)$, and $X \in C$ implies $X^{2}=0$. It follows that if $v \in V_{S}$, then $\theta\left(P_{v}\right)=P_{w}+X$ where $w \in V_{s}$ and similarly for $v \notin V_{s}$.

We are now ready to prove the theorem.

PROOF. Note that $\left.\widehat{\theta}\right|_{M_{Q_{s}}}$ is a homeomorphism since $M_{Q_{s}}$ is a countable set of points with the discrete topology. Now let

$$
i: \mathcal{T}^{+}\left(Q_{0}\right) \rightarrow \mathcal{T}^{+}(Q)
$$

be the inclusion map which is continuous. Furthermore, let

$$
P_{0}=\sum_{v \notin V_{s}} P_{v}
$$

and note that $\tilde{\theta}:=P_{0} \theta(i(X))$ is an automorphism of $\mathcal{T}^{+}\left(Q_{0}\right)$ onto the subalgebra $\mathcal{T}^{+}\left(Q_{0}\right) \subset \mathcal{T}^{+}(Q)$. As $\mathcal{T}^{+}\left(Q_{0}\right)$ has no sinks we know that $\tilde{\theta}$ is continuous $[5,3.15]$ and, hence, induces a homeomorphism on $M_{Q_{0}}$. However, note that $\left.\widehat{\theta}\right|_{M_{Q_{0}}}$ is the same as the map induced by $\tilde{\theta}$ on $M_{Q_{0}}$. So we know that $\left.\widehat{\theta}\right|_{M_{Q_{0}}}$ is continuous and, hence, $\theta$ induces a homeomorphism on $M_{Q}$.

We use this homeomorphism to define our first class of automorphisms. We say that an automorphism $\theta$ is component fixing if the induced homeomorphism $M_{\theta}$ fixes connected components of the maximal ideal space. In other words, if $X$ is a connected component of the maximal ideal space, then $M_{\theta}(X)=X$. We denote the set of all component fixing automorphisms of $\mathcal{T}^{+}(Q)$ by $\mathrm{CF}(Q)$.

TheOrem 3.7. The set $\mathrm{CF}(Q)$ is a normal subgroup of $\operatorname{Aut}\left(\mathcal{T}^{+}(Q)\right)$. Furthermore,

$$
\operatorname{Aut}\left(\mathcal{T}^{+}(Q)\right) / \mathrm{CF}(Q) \cong \mathrm{v}-\operatorname{Aut}(Q) \text { and } \operatorname{Aut}\left(\mathcal{T}^{+}(Q)\right) \cong \mathrm{CF}(Q) \oplus \operatorname{v-Aut}(Q) .
$$

ProOF. Clearly the identity automorphism is in $\operatorname{CF}(Q)$. If $\theta_{1}, \theta_{2} \in \operatorname{CF}(Q)$, then for a connected component $X \in M_{Q}$ we have $M_{\theta_{1} \circ \theta_{2}}(X)=M_{\theta_{1}}(X)=X$ and, hence, $\operatorname{CF}(Q)$ is closed with respect to products. Also if $M_{\theta^{-1}}(X)=X$, then $M_{\theta^{-1}}(X)=X$ and, hence, $\mathrm{CF}(Q)$ is a subgroup of the automorphism group. 
Now if $\theta \in \operatorname{Aut}\left(\mathcal{T}^{+}(Q)\right)$ and $\theta^{\prime} \in \mathrm{CF}(Q)$, then for a connected component $X \subseteq M_{Q}$ we have

$$
\begin{aligned}
M_{\theta \circ \theta^{\prime} \circ \theta^{-1}}(X) & =M_{\theta \circ \theta^{\prime}}\left(M_{\theta^{-1}}(X)\right) \\
& =M_{\theta}\left(M_{\theta^{-1}}(X)\right) \\
& =M_{\theta \circ \theta^{-1}}(X) \\
& =M_{\mathrm{id}}(X) \\
& =X .
\end{aligned}
$$

Denote by $S_{v}$ the closed subspace of $\ell^{2}(\mathcal{P}(Q))$ onto which $P_{v}$ is the projection. Since each of the $P_{v}$ are orthogonal and $\sum P_{v}=I$ we know that

$$
\ell^{2}(\mathcal{P}(Q))=\bigoplus_{v \in V(Q)} S_{v} .
$$

Now note that for a vertex $v, S_{v}$ is either one-dimensional (if $V$ does not support a nontrivial cycle) or $S_{v}$ has countably infinite dimension.

Let $\overline{\mathbb{B}_{n(v)}}$ be the connected component of $M_{Q}$ associated to the vertex $v$. Then note that if $\theta$ is an automorphism of $\mathcal{T}^{+}(Q)$, then the induced map on $M_{Q}$ will send $\overline{\mathbb{B}_{n(v)}}$ to some component $\overline{\mathbb{B}_{n\left(v^{\prime}\right)}}$ and note that $n(v)=n\left(v^{\prime}\right)$. For such $\theta$ note that the dimension of $S_{v}$ will be equal to the dimension of $S_{v^{\prime}}$ and, hence, there is an (not necessarily unique) isomorphism $\tau: S_{v} \rightarrow S_{v^{\prime}}$. For each pair $\left(v, v^{\prime}\right)$ associated to $\theta$ fix an isomorphism between $S_{v}$ and $S_{v^{\prime}}$ and call it $I_{v, v^{\prime}}$ and note that the map $U_{\theta}:=\sum_{v \in V(Q)} I_{v, v^{\prime}}$ is a unitary. We can assume without loss of generality that if $v=v^{\prime}$, then $I_{v, v^{\prime}}$ is the identity isomorphism. Further, if $\theta$ and $\theta^{\prime}$ are distinct automorphisms with the same pair $\left(v, v^{\prime}\right)$, then we assume (once again without loss of generality) that $I_{v, v^{\prime}}$ will be the same in both circumstances. We can do this beforehand by taking all possible pairs $\left(v, v^{\prime}\right)$ where $S_{v}$ and $S_{v^{\prime}}$ have the same cardinality and defining our $I_{v, v^{\prime}}$ without reference to the automorphism.

Now note that $\operatorname{Ad}\left(U_{\theta}\right)(x)=U_{\theta} x U_{\theta^{\prime}}$ is an automorphism of $\mathcal{T}^{+}(Q)$ and further a quick calculation tells us that $\operatorname{Ad}\left(U_{\theta}\right) \circ \theta \in \mathrm{CF}(Q)$.

In particular, we have a split exact sequence

$$
0 \longrightarrow \mathrm{CF}(Q) \longrightarrow \operatorname{Aut}\left(\mathcal{T}^{+}(Q) \stackrel{q}{\sum_{[\theta] \mapsto U_{\theta}} \operatorname{Aut}\left(\mathcal{T}^{+}\right.}(Q)\right) / \mathrm{CF}(Q) \longrightarrow 0,
$$

where $q$ is the quotient map. Of course, we still must verify that the map $[\theta] \mapsto U_{\theta}$ is well defined, but since the $I_{v, v^{\prime}}$ are independent of $\theta$ this is trivial. Further, following this map by $q$ will clearly induce the identity map on $\operatorname{Aut}\left(\mathcal{T}^{+}(Q)\right) / \mathrm{CF}(Q)$.

It only remains to verify that $\operatorname{Aut}\left(\mathcal{T}^{+}(Q)\right) / \mathrm{CF}(Q)$ is isomorphic to $\operatorname{v-Aut}(Q)$. We do this by making reference to a construction in [5]. There it is shown that given a directed graph algebra the directed graph $Q$ is an invariant of the algebra. 
In particular, they use $M_{Q}$ and the two-dimensional nest representations of $\mathcal{T}^{+}(Q)$ to construct the graph $Q$. One uses this construction to see that an automorphism $\theta$ will yield a graph automorphism of $Q$. Denote by $\tau_{\theta}$ the graph automorphism associated to $\theta$. We now claim that the map $U_{\theta} \mapsto\left(\tau_{U_{\theta}}\right)^{\prime}$ is an isomorphism between $\operatorname{v-Aut}(Q)$ and $\operatorname{Aut}\left(\mathcal{T}^{+}(Q)\right) / \mathrm{CF}(Q)$. That this map is a homomorphism follows from basic considerations of the map $U_{\theta}$ and its relationship to $\tau_{U_{\theta}}$. That the map is onto comes from constructing, given a fixed order-preserving automorphism of $Q$, call it $\tau$, an automorphism of $\mathcal{T}^{+}(Q)$ by letting $P_{v} \mapsto P_{\tau(v)}$ and $L_{e} \mapsto L_{\tau(e)}$ for all vertices $v$ and edges $e$.

We must finally verify that the homomorphism is one-to-one. However, note that if $\left(\tau_{U_{\theta_{1}}}\right)^{\prime}=\left(\tau_{U_{\theta_{2}}}\right)^{\prime}$, then $\tau_{U_{\theta_{1}} U_{\theta_{2}}^{-1}}$ fixes the vertices of $Q$ and, hence, $\left(\tau_{U_{\theta_{1}} U_{\theta_{2}}^{-1}}\right)^{\prime}$ is the identity map. The result now follows.

We note from the proof that the automorphisms which do not fix connected components of the maximal ideal space are implemented by unitaries in $B\left(\ell^{2}(\mathcal{P}(Q))\right)$ and, in particular, are continuous. We now look to analyze the group $\mathrm{CF}(Q)$.

\section{Gelfand fixing automorphisms}

Let $\theta$ be an automorphism with associated homeomorphism $M_{\theta}$ on $M_{Q}$. We say that $\theta$ is Gelfand fixing if $M_{\theta}$ is the identity homeomorphism.

Definition 4.1. Let $\mathrm{GF}(Q)$ denote the set of all continuous Gelfand fixing automorphisms of $\mathcal{T}^{+}(Q)$.

Note that if $\theta$ is Gelfand fixing, then, in particular, $\theta$ is component fixing. We see that more is true.

PROpOSITION 4.2. The set $\mathrm{GF}(Q)$ is a normal subgroup of $\operatorname{Aut}\left(\mathcal{T}^{+}(Q)\right)$.

PROOF. Clearly, the identity automorphism is an element of $\mathrm{GF}(Q)$. Further if $\theta \in \mathrm{GF}(Q)$, then $M_{\theta^{-1}}=\left(M_{\theta}\right)^{-1}=\mathrm{id}^{-1}=\mathrm{id}$ and, hence, $\operatorname{GF}(Q)$ is closed under inverses. Next note that if $\theta_{1}$ and $\theta_{2}$ are both in $\operatorname{GF}(Q)$, then

$$
M_{\theta_{1} \circ \theta_{2}}=M_{\theta_{1}} \circ M_{\theta_{2}}=\mathrm{id} \circ \mathrm{id}=\mathrm{id}
$$

and, hence, $\operatorname{GF}(Q)$ is a subgroup of $\operatorname{Aut}\left(\mathcal{T}^{+}(Q)\right)$.

Now if $\tau \in \operatorname{Aut}\left(\mathcal{T}^{+}(Q)\right)$ and $\theta \in \operatorname{GF}(Q)$, then

$$
\begin{aligned}
M_{\tau \circ \theta \circ \tau^{-1}} & =M_{\tau} \circ M_{\theta} \circ M_{\tau^{-1}} \\
& =M_{\tau} \circ M_{\theta} \circ\left(M_{\tau}\right)^{-1} \\
& =M_{\tau} \circ \mathrm{id} \circ\left(M_{\tau}\right)^{-1} \\
& =\mathrm{id}
\end{aligned}
$$

and, hence, $\tau \circ \theta \circ \tau^{-1}$ is an element of $\operatorname{GF}(Q)$. 
We now want to analyze the relationship between $\operatorname{CF}(Q)$ and $\operatorname{GF}(Q)$. Note that if a vertex supports no loop edges, then the component of $M_{Q}$ corresponding to $v$ is a one-point space. Hence, if $\theta$ is in $\mathrm{CF}(Q)$, then $\theta$ will be the identity homeomorphism on the component corresponding to $v$. We call such components trivial components. We see that the only components which can possibly give rise to automorphisms in $\mathrm{CF}(Q) \backslash \mathrm{GF}(Q)$ are the nontrivial components.

Now fix a component $X \in M_{Q}$ which corresponds to a vertex $v$ with $n$ loop edges supported on $v$. Let $F_{X}$ denote the set of those automorphisms $\theta \in \mathrm{CF}(Q)$ such that $M_{\theta}$ is the identity homeomorphism on $X$.

PROpOSITION 4.3. If $X$ is a component of $M_{Q}$, then $F_{X}$ is a normal subgroup of $\mathrm{CF}(Q)$.

Proof. Note that if $\theta \in F_{X}$, then $\left.M_{\theta^{-1}}\right|_{X}=\mathrm{id}=\left.M_{\theta}\right|_{X}$ and, hence, $F_{X}$ is closed with respect to inverses. Furthermore, if $\theta_{1}$ and $\theta_{2}$ are in $F_{X}$, then $\theta_{1} \circ \theta_{2}$ induces the trivial homeomorphism on $X$ and, hence, $F_{X}$ is closed with respect to composition. It follows that $F_{X}$ is a subgroup.

Now if $\theta \in F_{X}$ and $\sigma \in \mathrm{CF}(Q)$, then

$$
\begin{aligned}
M_{\left.\sigma \circ \theta \circ \sigma^{-1}\right|_{X}} & =\left.\left(M_{\sigma} \circ M_{\theta} \circ M_{\sigma^{-1}}\right)\right|_{X} \\
& =\left.\left.\left.M_{\sigma}\right|_{X} \circ M_{\theta}\right|_{X} \circ M_{\sigma^{-1}}\right|_{X} \\
& =\left.\left.M_{\sigma}\right|_{X} \circ M_{\sigma^{-1}}\right|_{X} \\
& =\mathrm{id}
\end{aligned}
$$

and, hence, $F_{X}$ is normal.

Note that if $X$ is a trivial component, then $F_{X}=\mathrm{CF}(Q)$. Furthermore, if $X$ is the only nontrivial component of $M_{Q}$, then $F_{X}=\mathrm{CF}(Q)$.

PROposition 4.4. Suppose that $\left\{X_{i}\right\}_{i=1}^{\infty}$ are the mutually disjoint set of all nontrivial components of $M_{Q}$, then

$$
\bigcap_{i=1}^{\infty} F_{X_{i}}=\mathrm{GF}(Q) .
$$

Proof. Clearly every automorphism in $\mathrm{GF}(Q)$ will fix the components $X_{i}$ and, hence, $\mathrm{GF}(Q) \subseteq \bigcap_{i=1}^{\infty} F_{X_{i}}$. Now if $\theta \in \bigcap_{i=1}^{\infty} F_{X_{i}}$, then $M_{\theta}$ will be the identity on every component and, hence, $\theta \in \mathrm{GF}(Q)$.

Now for a nontrivial component $X \subseteq M_{Q}$ let $G_{X}$ be the normal subgroup $\bigcap_{Y \neq X} F_{Y}$, where $Y$ ranges over the set of nontrivial components in $M_{Q}$. Note that $G_{X}=\mathrm{CF}(Q) / F_{X}$ via the natural map. If we analyze the situation of a single graph with $n$ vertices, as was done in the weakly closed case in [3], we can actually figure out what $G_{X} / \mathrm{GF}(Q)$ looks like where $X$ is a nontrivial component of $M_{Q}$. We remind the reader that $\mathbb{B}_{n}$ denotes the open unit ball of $\mathbb{C}^{n}$ and let Aut $\left(\mathbb{B}_{n}\right)$ denote the conformal automorphisms of $\mathbb{B}_{n}$. 
THEOREM 4.5. Let $X$ be a component corresponding to the vertex $v$ which supports $n$ loop edges. Then there is a short exact sequence

$$
0 \rightarrow \mathrm{GF}(Q) \rightarrow G_{X} \rightarrow \operatorname{Aut}\left(\mathbb{B}_{n}\right) \rightarrow 0 .
$$

This exact sequence does not split. However, there is a group of unitaries $U_{X}$ which implement automorphisms in $G_{X}$ and such that there is a group isomorphism of $U_{X}$ onto $\operatorname{Aut}\left(\mathbb{B}_{n}\right)$.

PROOF. For this proof we take each subalgebra which is generated by a single vertex and all of the loops attached to that vertex and use the arguments of [3]. The unitaries are then applied to the whole algebra treating each component separately.

The following corollary is now an easy computation.

COROllary 4.6. The group $\mathrm{CF}(Q) / \mathrm{GF}(Q)$ is homeomorphic to

$$
\oplus\left\{G_{X} \mid X \subseteq M_{Q}, X \text { nontrivial connected component }\right\}
$$

PROOF. Note that if $X$ and $Y$ are disjoint connected components of $M_{Q}$, then $G_{X} \cap G_{Y}=\mathrm{GF}(Q)$, by Proposition 4.4. Furthermore, if $\theta \in \mathrm{CF}(Q)$ let $u_{X}$ denote the unitary in $U_{X}$ which implements the automorphism in $\left(\mathrm{CF}(Q) / F_{X}\right) / \mathrm{GF}(X)$ which is isomorphic via the natural map to $G_{X}$. Now define a unitary $v_{X}$ in $B\left(\ell^{2}(Q)\right)$ by letting $v_{X}$ act as the identity on the subspace $\oplus\left\{S_{v} \mid v\right.$ does not correspond to $\left.X\right\}$ and letting $v_{X} \mid S_{w}=u_{X}$, where $w$ is the vertex corresponding to $X$. Then note that

$$
\theta=\prod_{X \in M_{Q}} \operatorname{Ad}\left(v_{X}\right) .
$$

As each group $G_{X}$ is normal the result now follows.

In the remainder of the paper we look at the group $\mathrm{GF}(Q)$. We can see, by using the unitaries we have already discussed, that if $\theta$ is an automorphism of $\mathcal{T}^{+}(Q)$, then $\theta=\theta_{1} \circ \theta_{2}$ where $\theta_{2}$ is continuous and $\theta_{1} \in \mathrm{GF}(Q)$. This is used later in discussing continuity of automorphisms. In particular, $\theta$ is continuous if and only if $\theta_{1}$ is continuous.

\section{Factoring automorphisms through subalgebras}

We use ideas from [4] to look at automorphisms. In particular, let $\pi: \mathcal{T}^{+}(Q) \rightarrow A$ be a completely contractive representation of $\mathcal{T}^{+}(Q)$ onto a subalgebra $A \subseteq \mathcal{T}^{+}(Q)$. We say that an automorphism $\theta$ of $\mathcal{T}^{+}(Q)$ factors through $A$ via $\pi$ if there is an automorphism $\theta_{A}$ of $A$ such that $\theta_{A}(a)=(\pi \circ \theta)(a)$ for all $a \in A$.

PROPOSITION 5.1. Let $\pi: \mathcal{T}^{+}(Q) \rightarrow$ A be a completely contractive representation of $\mathcal{Q}$ into a subalgebra $A \subseteq \mathcal{T}^{+}(Q)$. Then an automorphism $\theta \in \operatorname{Aut}\left(\mathcal{T}^{+}(Q)\right.$ factors through $A$ via $\pi$ if and only if $\theta$ induces an automorphism on $\operatorname{ker} \pi$. 
Proof. Clearly if $\theta$ factors through $A$, via $\pi$, then the image of $\operatorname{ker} \pi$ under $\theta$ is a subset of ker $\pi$ otherwise $\theta_{A}$ will not be well defined. Now if the image of ker $\pi$ under $\theta$ is equal to $\operatorname{ker} \pi$, then the map $\theta_{A}: A \rightarrow A$ is given by $\theta_{A}(a)=\pi \circ \theta(b)$, where $b$ is any element of $\mathcal{T}^{+}(Q)$ such that $\pi(B)=a$, and $\theta_{A}$ is well defined. Furthermore, $\theta_{A}$ will be onto since $\theta$ and $\pi$ are onto, and $\theta_{A}$ will be one-to-one since $\pi \circ \theta(x)=$ $\pi \circ \theta(y)$ if and only if $\theta(x-y) \in \theta(\operatorname{ker} \pi)$ which implies $x-y \in \operatorname{ker} \pi$.

In this language the results of our preceding section can be restated. If $\theta \in \mathrm{CF}(Q)$, then $\theta$ factors through the subalgebra

$$
\bigoplus_{v: n(v) \geq 1} A_{n(v)}
$$

via the homomorphism generated by sending every nonloop edge and every vertex not supporting a loop edge to zero and leaving loop edges and loop supporting vertices alone. The normal subgroup $\operatorname{GF}(Q)$ are those automorphisms which are quasi-inner, in the language of [3]. In this case, however, because there may be edges which are not loops the normal subgroup $\mathrm{GF}(Q)$ may contain quasi-inner automorphisms which are clearly not inner. We want to discuss such automorphisms now. To do so we look at how automorphisms factor through two types of subalgebras.

The first class of subalgebras are those generated by subgraphs given by a pair of vertices $v_{1}, v_{2}$ and all edges $e$ with $s(e)=v_{2}$ and $r(e)=v_{1}$. Note that this subalgebra will be isomorphic to the graph algebra $\mathcal{T}^{+}\left(T_{2}^{(n)}\right)$ where $T_{2}^{(n)}$ is the graph with two vertices and $n$ directed edges between them, all with the same source. If $n=1$ this algebra is isomorphic to the upper triangular $2 \times 2$ matrices.

The second class of subalgebras are given by choosing a nontrivial cycle in $Q$, call it $w=e_{n} e_{n-1} \cdots e_{2} e_{1}$, such that $n \geq 2$. We also assume that $s\left(e_{i}\right) \neq s\left(e_{j}\right)$ for all $i \neq j$. In this case the subgraph with vertex set $\left\{s\left(e_{i}\right)\right\}$ and edge set $\left\{e_{i}\right\}$ generates a subalgebra isomorphic to $A\left(\mathcal{C}_{n}\right)$ where $\mathcal{C}_{n}$ is a cycle of length $n \geq 2$.

Let us now define our first representation. Let $v_{1}$ and $v_{2}$ be distinct vertices in $Q$ and let $\left\{e_{i}\right\}_{i=1}^{n}$, be the set of edges with source being equal to $v_{2}$ and range being equal to $v_{1}$. We now want to define a representation $\pi_{v_{1}, v_{2}}: \mathcal{T}^{+}(Q) \rightarrow \mathcal{T}^{+}\left(T_{2}^{(n)}\right)$. Begin by sending $P_{v}$ to 0 for all vertices $v \neq v_{i}$ for any $i$, and $L_{e}$ to 0 for all edges $e \neq e_{j}$ for any $j$. Then map $P_{v_{1}}$ to the projection of the range vertex in $\mathcal{T}^{+}\left(T_{2}^{(n)}\right)$ and $P_{v_{2}}$ to the other vertex projection in $\mathcal{T}^{+}\left(T_{2}^{(n)}\right)$. Lastly, map $L_{e_{i}}$ to the distinct partial isometries in $\mathcal{T}^{+}\left(T_{2}^{(n)}\right)$ corresponding to the edges in $T_{2}^{(n)}$. That this map extends to a completely contractive representation of $\mathcal{T}^{+}(Q)$ follows from [2, Proposition 1.3] since the left regular representation of $\mathcal{T}^{+}\left(T_{2}^{(n)}\right)$ is pure. An interesting thing to note is that the representation is unital.

We now claim that if $\theta \in \mathrm{GF}(Q)$, then $\theta$ factors through $\mathcal{T}^{+}\left(T_{2}^{(n)}\right)$ via the defined representations.

Proposition 5.2. If $\theta \in \mathrm{GF}(Q)$, then given two distinct vertices $v_{1}$ and $v_{2}$ in $V(Q)$, $\theta$ factors through $\mathcal{T}^{+}\left(T_{2}^{(n)}\right)$ via $\pi_{v_{1}, v_{2}}$. 
PROOF. Let $a \in \operatorname{ker} \pi_{v_{1}, v_{2}}$, then $a$ is in the ideal generated by $\left\{P_{v} \mid v \neq v_{1}, v_{2}\right\}$ and $\left\{L_{e} \mid r(e) \neq v_{1}\right.$, or $\left.s(e) \neq v_{2}\right\}$. However, note that as $\theta$ is in $\operatorname{GF}(Q)$ we know that $\theta\left(P_{v}\right) \in \operatorname{ker} \pi_{v_{1}, v_{2}}$ for all vertices $v \neq v_{i}$. It follows that $\theta\left(L_{e}\right) \in \operatorname{ker} \pi_{v_{1}, v_{2}}$ for all edges $e$ with $r(e) \neq v_{i}$ or $s(e) \neq v_{i}$.

Now if $e$ is an edge with $r(e)=v_{1}$, then $\pi_{v_{1}, v_{2}}\left(\theta\left(L_{e}\right)\right)=0$ since $\theta$ is in $\operatorname{GF}(Q)$. Similarly if $s(e)=v_{2}$, then $\theta\left(L_{e}\right) \in \operatorname{ker} \pi_{v_{1}, v_{2}}$ and, hence, $\theta\left(\operatorname{ker} \pi_{v_{1}, v_{2}}\right) \subseteq \operatorname{ker} \pi_{v_{1}, v_{2}}$. As the same argument tells us that $\theta^{-1}\left(\operatorname{ker} \pi_{v_{1}, v_{2}}\right) \subseteq \operatorname{ker} \pi_{v_{1}, v_{2}}$ we obtain that $\theta$ is an automorphism of ker $\pi_{v_{1}, v_{2}}$ and, hence, $\theta$ factors through $\pi_{v_{1}, v_{2}}$.

In fact more is true. It is not hard to see that if $\theta \in \mathrm{CF}(Q)$, then $\theta$ factors through $\mathcal{T}^{+}\left(T_{2}^{(n)}\right)$. However, when we factor $\theta$ in this way we lose all of the information about what $\theta$ does to the components of $M_{Q}$ and, hence, we focus only on those automorphisms in $\mathrm{GF}(Q)$.

\section{Automorphisms of $\mathcal{T}^{+}\left(T_{2}^{(n)}\right)$}

We now analyze automorphisms of the graph algebra $\mathcal{T}^{+}\left(T_{2}^{(n)}\right)$ with an eye toward understanding how automorphisms of general quiver algebras factor through these subalgebras. We begin by fixing some notation. Let $P_{1}$ and $P_{2}$ denote the projections associated to the range and source projections, respectively. Let $L_{i}$ denote the partial isometry associated to the $i$ th edge. Note that $P_{1} L_{i}=L_{i}=L_{i} P_{2}$ for all $i$. Further $L_{i} L_{j}=0$ for all $i \neq j$. It follows that every element of $\mathcal{T}^{+}\left(T_{2}^{(n)}\right)$ can be written as

$$
\alpha P_{1}+\sum_{i=1}^{n} \alpha_{i} L_{i}+\beta P_{2}
$$

where $\alpha, \beta$ and $\alpha_{i}$ are complex numbers.

LeMma 6.1. Let $\theta: \mathcal{T}^{+}\left(T_{2}^{(n)}\right) \rightarrow \mathcal{T}^{+}\left(T_{2}^{(n)}\right)$ be an automorphism. Then there exist $\alpha_{i}$ such that $\theta\left(P_{1}\right)=P_{1}+\sum_{i=1}^{n} \alpha_{i} L_{i}$ and $\theta\left(P_{2}\right)=P_{2}-\sum_{i=1}^{n} \alpha_{i} L_{i}$.

Proof. We know that $\theta\left(P_{1}\right)=\alpha P_{1}+\sum_{i=1}^{n} \alpha_{i} L_{i}+\beta P_{2}$ for some $\alpha, \beta$, and $\alpha_{i}$. Now $\theta\left(P_{1}^{2}\right)=\theta\left(P_{1}\right)$ and hence

$$
\alpha P_{1}+\sum_{i=1}^{N} \alpha_{i} L_{i}+\beta P_{2}=(\alpha)^{2} P_{1}+(\alpha+\beta) \sum_{i=1}^{n} \alpha_{i} L_{i}+(\beta)^{2} P_{2}
$$

It follows that $\alpha$ and $\beta$ are idempotents, hence zero or one. If $\alpha$ and $\beta$ are both one, then $\sum_{i=1} \alpha_{i} L_{i}=0$ and, hence, $\theta\left(P_{1}\right)=P_{1}+P_{2}$ which is the identity which yields a contradiction.

Note that $\alpha$ cannot be zero or otherwise we would have an automorphism which does not fix components of the maximal ideal space, which is not possible with this graph. It follows that $\alpha=1$ and $\beta=0$. The first part of the result is established. Next note that the same calculation for $\theta\left(P_{2}\right)$ and noting that $P_{1}+P_{2}=$ id we obtain that $\theta\left(P_{2}\right)=P_{2}-\sum_{i=1}^{\infty} \alpha_{i} L_{i}$ and the result follows. 
We now focus on what an automorphism can do to the partial isometries associated to edges.

LEMMA 6.2. Let $\theta$ be an automorphism of $\mathcal{T}^{+}\left(T_{2}^{(n)}\right)$ with $\theta\left(P_{1}\right)=P_{1}+\sum_{i=1}^{n} \alpha_{i} L_{i}$. There exists $\beta_{i, j}$ such that $\theta\left(L_{i}\right)=\sum_{j=1}^{n} \beta_{i, j} L_{j}$.

ProOf. Assume that $\theta\left(L_{i}\right)=a_{i} P_{1}+\sum_{j=1}^{n} \alpha_{i, j} L_{j}+b_{i} P_{2}$. We know that $\theta\left(L_{i}\right)=$ $\theta\left(P_{1}\right) \theta\left(L_{i}\right) \theta\left(P_{2}\right)$ and, hence,

$$
\begin{aligned}
a_{i} P_{1}+\sum_{j=1}^{n} \alpha_{i, j} L_{j}+b_{i} P_{2}= & \left(P_{1}+\sum_{i=1}^{n} \alpha_{i} L_{i}\right) \theta\left(L_{i}\right)\left(P_{2}-\sum_{i=1}^{n} \alpha_{i} L_{i}\right) \\
= & \left(P_{1}+\sum_{i=1}^{n} \alpha_{i} L_{i}\right)\left(a_{i} P_{1}+\sum_{j=1}^{n} b_{i, j} L_{j}+b_{i} P_{2}\right) \\
& \times\left(P_{2}-\sum_{i=1}^{n} \alpha_{i} L_{i}\right) \\
= & -a_{i} \sum_{i=1}^{n} \alpha_{i} L_{i}+\sum_{j=1}^{n} b_{i, j} L_{j}+\sum_{i=1}^{n} \alpha_{i} b_{i} L_{i} .
\end{aligned}
$$

Letting $\beta_{i, i}=-a_{i} \sum_{i=1}^{n} \alpha_{i}+b_{i, i}+\sum_{i=1}^{n} \alpha_{i} b_{i}$ and $\beta_{i, j}=b_{i, j}$ for $i \neq j$ the result follows.

So, in effect, every automorphism of $\mathcal{T}^{+}\left(T_{2}^{(n)}\right)$ has associated to it an $n$-tuple $\left(\alpha_{1}, \alpha_{2}, \ldots, \alpha_{n}\right)$ and an invertible linear map $L_{i} \mapsto \sum_{j=1}^{n} \beta_{i, j} L_{j}$. It is clear that every such tuple and invertible linear map gives rise to an automorphism of $\mathcal{T}^{+}\left(T_{2}^{(n)}\right)$. We write the linear map as a matrix in the obvious way. Note that the automorphism will be continuous if and only if the matrix gives rise to a continuous linear operator. If $n$ is finite this is always true. However, in the case of infinite directed edges between two vertices the matrix may not give rise to a continuous linear operator. This fact, on a certain level, is where continuity of automorphisms of $\mathcal{T}^{+}(Q)$ can break down when there is a sink and a source in the graph. The next question that arises is which of these automorphisms are inner. To discuss this we need to describe the invertible elements of $\mathcal{T}^{+}\left(T_{2}^{(n)}\right)$.

Proposition 6.3. An element $x:=\alpha P_{1}+\sum_{i=1}^{n} \alpha_{i} L_{i}+\beta P_{2}$ is invertible if and only if $\alpha$ and $\beta$ are invertible; if so, $x^{-1}=\alpha^{-1} P_{1}-\alpha^{-1} \beta^{-1} \sum_{i=1}^{n} \alpha_{i} L_{i}+\beta^{-1} P_{2}$.

PRoOF. If $x$ is invertible, then there exists $y=a P_{1}+\sum_{i=1}^{n} a_{i} L_{i}+b P_{2}$ such that $x y=P_{1}+P_{2}$. However, note that $x y=\alpha a P_{1}+\sum_{i=1}^{n}\left(\beta a_{i}+a \alpha_{i}\right) L_{i}+\beta b P_{2}$. The result follows by setting $\beta a_{i}=-a \alpha_{i}$ and solving for $a_{i}$.

A simple calculation now yields the following description of inner automorphisms of $\mathcal{T}^{+}\left(T_{2}^{(n)}\right)$. 
PROPOSITION 6.4. An automorphism $\theta$, of $\mathcal{T}^{+}\left(T_{2}^{(n)}\right)$, is inner if and only if the matrix of the associated linear map is a nonzero multiple of the identity.

PROOF. For the forward direction we need only see what the inner automorphism $\theta$ does to $L_{i}$. So let $x=\alpha P_{1}+\sum_{i=1}^{n} \alpha_{i} L_{i}+\beta P_{2}$ be invertible and note that

$$
\begin{aligned}
x L_{i} x^{-1} & =\left(\alpha P_{1}+\sum_{i=1}^{n} \alpha_{i} L_{i}+\beta P_{2}\right) L_{i}\left(\alpha^{-1} P_{1}-\alpha^{-1} \beta^{-1} \sum_{i=1}^{n} \alpha_{i} L_{i}+\beta^{-1} P_{2}\right) \\
& =\beta \alpha^{-1} L_{i}
\end{aligned}
$$

which is independent of $i$.

Assume that $\theta\left(P_{1}\right)=P_{1}+\sum_{i=1}^{n} a_{i} L_{i}$ and if the associated linear map is $\lambda I_{n}$ with $\lambda \neq 0$, then let $x=\lambda^{-1} P_{1}+\lambda^{-1} \sum_{i=1}^{n} a_{i}+P_{2}$, so $x$ is invertible and $x L_{i} x^{-1}=\lambda L_{i}$. In addition $x \theta\left(P_{1}\right) x^{-1}=P_{1}+\sum_{i=1}^{n} a_{i} L_{i}$ and, hence, $x$ induces the same map as $\theta$ and the result follows.

We denote the normal subgroup of $\mathrm{GL}_{n}(\mathbb{C})$ given by multiples of the identity as $\lambda I_{n}$. This subgroup is normal because every element of $\lambda I_{n}$ commutes with every element of $\mathrm{GL}_{n}(\mathbb{C})$.

PROpOSITION 6.5. Every automorphism $\theta$ of $\mathcal{T}^{+}\left(T_{2}^{(n)}\right)$ can be written as $\theta_{1} \circ \theta_{2}$, where $\theta_{1}$ is inner and $\theta_{2}\left(P_{i}\right)=P_{i}$.

Proof. Assume that $\theta\left(P_{1}\right)=P_{1}+\sum_{i=1}^{n} \alpha_{i} L_{i}$. Then let $x=P_{1}+\sum_{i=1}^{n} \alpha_{i} L_{i}+P_{2}$. Note that $x$ is invertible. Now let $\theta_{1}=\operatorname{Ad}(x)$ and let $\theta_{2}=\theta_{1}^{-1} \circ \theta$. Note that $\theta_{1}$ is inner and $\theta_{2}\left(P_{1}\right)=\theta_{1}^{-1} \circ \theta\left(P_{1}\right)=\theta_{1}^{-1}\left(P_{1}+\sum_{i=1}^{n} \alpha_{i} L_{i}\right)=P_{1}$ and the result follows.

It is clear that $\theta_{1}$ is unique. However, it is not the case that $\theta_{2}$ is not inner. We do know, however, that if $\theta_{2}$ is inner, then the invertible mapping giving rise to $\theta_{2}$ is of the form $\lambda_{1} P_{1}+\lambda_{2} P_{2}$. To finish this description denote by $\operatorname{Inn}\left(\mathcal{T}^{+}\left(T_{2}^{(n)}\right)\right)$ the normal subgroup of $\operatorname{Aut}\left(\mathcal{T}^{+}\left(T_{2}^{(n)}\right)\right)$ of all the inner automorphisms. Further, denote by $\operatorname{Out}\left(\mathcal{T}^{+}\left(T_{2}^{(n)}\right)\right)$ the $\operatorname{group} \operatorname{Aut}\left(\mathcal{T}^{+}\left(T_{2}^{n}\right)\right) / \operatorname{Inn}\left(\mathcal{T}^{+}\left(T_{2}^{(n)}\right)\right)$. For $\theta \in \operatorname{Aut}\left(\mathcal{T}^{+}\left(T_{2}^{(n)}\right)\right)$ we denote its equivalence class in $\operatorname{Out}\left(\mathcal{T}^{+}\left(T_{2}^{(n)}\right)\right)$ by $[\theta]$.

THEOREM 6.6. The group $\operatorname{Out}\left(\mathcal{T}^{+}\left(T_{2}^{(n)}\right)\right)$ is isomorphic to $\mathrm{GL}_{n}(\mathbb{C}) / \lambda I_{n}$.

PROOF. By the previous proposition we know that given an automorphism $\theta$ there is associated to $[\theta]$ a unique element of $\mathrm{GL}_{n}(\mathbb{C})$. This is because after fixing the vertices, as in the proposition, the element of $\mathrm{GL}_{n}(\mathbb{C})$ associated to $\theta$ must be unique. We can now assume that $[\theta]$ comes from an automorphism which fixes the $P_{i}$. Note that $\lambda I_{n}$ is a normal subgroup which is in one-to-one correspondence with all of the inner automorphisms which fix the $P_{i}$. The result now follows.

We know that every Gelfand fixing automorphism of $\mathcal{T}^{+}(Q)$ will factor through the various copies of $\mathcal{T}^{+}\left(T_{2}^{(n)}\right)$. We see, however, that we cannot take arbitrary 
automorphisms of $\mathcal{T}^{+}\left(T_{2}^{(n)}\right)$ and piece them together. Norm considerations will force restrictions on the way they fit together.

\section{Automorphisms of $\mathcal{T}^{+}(Q)$ factoring through $\mathcal{T}^{+}\left(T_{2}^{(n))}\right)$}

It is known that if $Q$ is a graph which is infinite in one direction and $\theta: \mathcal{T}^{+}(Q) \rightarrow$ $\mathcal{T}^{+}\left(Q^{\prime}\right)$ is an algebraic isomorphism, then $\theta$ is automatically continuous, see [5]. In particular, every automorphism of $\mathcal{T}^{+}(Q)$ is automatically continuous. We now use information on how an automorphism factors through $T^{+}\left(T_{2}^{(n)}\right)$ to discuss the general question of continuity.

Let $Q$ be a directed graph. We say that $\left(v_{1}, v_{2}, \ldots v_{n}\right)$ is a finite path of vertices in $Q$ if for $1 \leq i \leq n-1$ there exists an edge $e_{i}$ such that $s\left(e_{i}\right)=v_{i+1}$ and $r\left(e_{i}\right)=v_{i}$. Unless stated otherwise such a path of vertices will be denoted by $V$ and its length will be $V_{n}$. For such a path of vertices let $n(i)$ denote the number of directed edges with $s(e)=v_{i+1}$ and $r(e)=v_{i}$.

If $\theta \in \mathrm{GF}(Q)$, then for every finite path of vertices, $V$ in $Q, \theta$ will factor through $\mathcal{T}^{+}\left(T_{2}^{\left(n_{i}\right)}\right)$ for all $1 \leq i \leq V_{n}-1$. Now associated to each pair of adjacent vertices $v_{i}, v_{i+1} \in V$ there is from the previous section a matrix $M_{i} \in \mathrm{GL}_{n(i)}(\mathbb{C})$.

LEMMA 7.1. Let $\theta$ be an automorphism of $\mathcal{T}^{+}(Q)$. If $\theta$ is continuous, then

$$
M:=\sup \left\{\left\|M_{1} M_{2} \cdots M_{V_{n}-1}\right\| V \text { is a finite path of vertices in } Q\right\}<\infty \text {. }
$$

PROOF. Certainly $\|\theta\| \geq M$ and the result follows.

REMARK 7.2. Note that if $v_{1}, v_{2}, \ldots v_{n}$ are consecutive edges in a cycle, then

$$
M_{\left(v_{n}, v_{n-1}\right)} M_{\left(v_{n-1}, v_{n-2}\right)} \cdots M_{\left(v_{2}, v_{1}\right)} M_{\left(v_{1}, v_{n}\right)}
$$

must have norm less than or equal to one, otherwise continued finite paths around the cycle would lead to an unbounded sequence of matrices which is excluded by the proposition.

Let $\mathrm{T}_{2} \operatorname{Inn}(Q)$ denote the set of those automorphisms in $\mathrm{GF}(Q)$ such that, for every $V$, a finite path of vertices in $Q$ and every $1 \leq i \leq V_{n}-1$ the matrices $M_{i}$ will be in $\lambda \cdot I_{n(i)}$.

PROPOSITION 7.3. If $Q$ is a directed graph, then $\mathrm{T}_{2} \operatorname{Inn}(Q)$ is a normal subgroup of $\mathrm{GF}(Q)$.

PRoOF. If $\theta_{1}, \theta_{2} \in \mathrm{T}_{2} \operatorname{Inn}(Q)$, then the matrices associated to $\theta_{1} \circ \theta_{2}$ will just be the product of the matrices associated to $\theta_{1}$ and $\theta_{2}$, respectively. Now the product of two matrices in $I_{n(i)}$ will still be in $I_{n(i)}$ and, hence, $\mathrm{T}_{2} \operatorname{Inn}(Q)$ will be closed under composition. That $\mathrm{T}_{2} \operatorname{Inn}(Q)$ is closed under inverses is trivial and, hence, $\mathrm{T}_{2} \operatorname{Inn}(Q)$ is a subgroup of $\operatorname{GF}(Q)$. 
Next, let $\theta_{1} \in \mathrm{T}_{2} \operatorname{Inn}(Q)$ and $\theta_{2} \in \mathrm{GF}(Q)$, then for every pair of adjacent vertices $\theta_{2}$ will have a matrix in $\mathrm{GL}_{n}(\mathbb{C})$ associated to it. However, the matrix associated to $\theta_{1}$ will be a multiple of the identity and, hence, will commute with the matrices associated to $\theta_{2}$. It follows that $\theta_{2} \circ \theta_{1} \circ \theta_{2}^{-1} \in \mathrm{T}_{2} \operatorname{Inn}(Q)$ and, hence, $\mathrm{T}_{2} \operatorname{Inn}(Q)$ will be normal.

Let GL $(Q)$ denote the set of uniformly bounded finite sequences of automorphism matrices associated to finite paths in $Q$. We denote by $\lambda \mathrm{GL}(Q)$ the normal subgroup of $\operatorname{GL}(Q)$ given by finite sequences in which each matrix in the sequence is a multiple of the identity on the appropriate space.

Proposition 7.4. Given a directed graph $Q$ the groups $\operatorname{GF}(Q) / \mathrm{T}_{2} \operatorname{Inn}(Q)$ and $\mathrm{GL}(Q) / \lambda \mathrm{GL}(Q)$ are isomorphic.

ProOF. We first show that every sequence of matrices in $\operatorname{GL}(Q)$ gives rise to an automorphism in $\mathrm{GF}(Q)$. The remainder is then just a restatement of Proposition 7.3 in the context of general graph automorphisms by looking at the factorizations through $T_{2}$.

So let $\left\{M_{(v, w)}\right\}_{(v, w) \in V \times V}$ denote a set of matrices in $\operatorname{GL}(Q)$. We want to show the existence of an automorphism which gives rise to this set of matrices. Define $\theta\left(P_{v}\right)=P_{v}$ for all vertices $v \in V(Q)$. Define $\theta\left(L_{e}\right)=L_{e}$ for all loops $e \in E(Q)$. Lastly, for every pair of vertices $(v, w)$ place an ordering on the set $E_{(v, w)}$ and apply $M_{(v, w)}$ to $E_{(v, w)}$ to calculate what $\theta\left(L_{e}\right)$ will do for $e \in E_{(v, w)}$. Now $\theta$ will extend to an automorphism in $\operatorname{GF}(Q)$ with matrix set $\left\{M_{(v, w)}\right\}$, hence, $\theta$ will be a continuous automorphism in $\mathrm{GF}(Q)$.

THEOREM 7.5. For $Q$ a directed graph, $\mathrm{GF}(Q)$ splits as

$$
\mathrm{T}_{2} \operatorname{Inn}(Q) \oplus\left(\mathrm{GF}(Q) / \mathrm{T}_{2} \operatorname{Inn}(Q)\right)
$$

PROOF. We know that we have the short exact sequence

$$
0 \longrightarrow \mathrm{T}_{2} \operatorname{Inn}(Q) \longrightarrow \mathrm{GF}(Q) \longrightarrow \mathrm{GF}(Q) / \mathrm{T}_{2} \operatorname{Inn}(Q) \longrightarrow 0 \text {. }
$$

We only need to show that it splits. Now, for every adjacent pair of vertices, put an ordering on the edges between them. Note that $[\theta] \in \mathrm{GF}(Q) / \mathrm{T}_{2} \operatorname{Inn}(Q)$ has associated to each adjacent pair of vertices $(v, w)$ a matrix $M_{(v, w)}$. Now for $[\theta] \in \mathrm{GF}(Q) / \mathrm{T}_{2} \operatorname{Inn}(Q)$ define $j([\theta])$ to be the automorphism induced by sending $P_{i}$ to $P_{i}, L_{e}$ to $L_{e}$ if $e$ is a loop edge, and for $f_{i}$ between the adjacent pair $(v, w)$ have the automorphism send $L_{f_{i}}$ to the linear combination of the edges between $v$ and $w$ given by applying $M_{(v, w)}$ to the column matrix for $L_{f_{i}}$ in the natural way.

It is easy to see that $j([\theta])$ defines an automorphism of $\mathcal{T}^{+}(Q)$ and further that $j([\theta]) \in \mathrm{GF}(Q)$. A quick calculation tells one that $q \circ j$ is the identity on $\mathrm{GF}(Q) / \mathrm{T}_{2} \operatorname{Inn}(Q)$ where $q$ is the natural quotient. The result now follows. 


\section{The group $T_{2} \operatorname{Inn}(Q)$}

We now want to analyze those automorphisms in $\mathrm{T}_{2} \operatorname{Inn}(Q)$. Once again we factor these automorphisms through a subalgebra. In this case, however, the subalgebra will be those corresponding to directed cycles. Recall that for a positive integer $k, \mathcal{C}_{k}$ is the graph with $k$ vertices and $k$ edges forming a single directed cycle. Let $w=e_{1} e_{2} \cdots e_{n}$, with $n \geq 2$, denote a cycle in $Q$ such that $r\left(e_{i}\right) \neq r\left(e_{j}\right)$ for $i \neq j$. We call such a cycle a proper cycle. Let $W$ denote the set of all proper cycles and note that every $w \in W$ gives rise to a finite sequence of vertices in $Q$. Let $w(n)$ denote the length of the cycle $w \in W$. Note that such a $w$ yields a subalgebra of $\mathcal{T}^{+}(Q)$ which is isomorphic, via graph isomorphism, to $\mathcal{T}^{+}\left(\mathcal{C}_{w(n)}\right)$; call this algebra $\mathcal{T}^{+}\left(\mathcal{C}_{w}\right)$. Fixing a proper cycle in $Q$ we now define a representation of $\pi_{w}: \mathcal{T}^{+}(Q) \rightarrow \mathcal{T}^{+}\left(\mathcal{C}_{w(n)}\right)$. This follows by sending $P_{v}$ and $L_{e}$ to 0 whenever $v$ or $e$ does not support $w$ and sending $P_{v}$ and $L_{e}$ to the appropriate projection, or partial isometry, in the cycle algebra. One then extends this map to a completely contractive unital representation of $\mathcal{T}^{+}(Q)$.

Unlike the automorphisms which factor through $\mathcal{T}^{+}\left(\mathrm{T}_{2}^{(n)}\right)$ it is not the case that every Gelfand fixing automorphism will factor through $\mathcal{T}^{+}\left(\mathcal{C}_{n}\right)$. Instead we have the following proposition.

PROpOSITION 8.1. If an automorphism $\theta \in \mathrm{GF}(Q)$ is in $\mathrm{T}_{2} \operatorname{Inn}(Q)$, then $\theta$ factors through the subalgebra $T^{+}\left(\mathcal{C}_{w}\right)$ for every cycle $w \in W$.

Proof. Assume that $P_{v} \in \operatorname{ker} \pi_{w}$, then note that, as $\theta$ is in $\operatorname{GF}(Q), \theta\left(P_{v}\right) \in \operatorname{ker} \pi_{w}$. Now if $L_{e} \in \operatorname{ker} \pi_{w}$, then there are two cases. If $r(e)=s(e)$, then $\theta\left(L_{e}\right) \in \operatorname{ker} \pi_{w}$ as $\theta\left(P_{r(e)}\right)=\theta\left(P_{s(e)}\right)$. Otherwise, we assume that $r(e) \neq s(e)$ in which case $\pi_{(r(e), s(e))}\left(\theta\left(L_{e}\right)\right)=\lambda L_{e}$. It follows that $\theta\left(L_{e}\right) \in \operatorname{ker} \pi_{w}$ if and only if $L_{e} \in \operatorname{ker} \pi_{w}$. Now as ker $\pi_{w}$ is generated by those $P_{v}$ and $L_{e}$ in ker $\pi_{w}$ the result follows.

That the converse is not true follows by looking at a graph with no cycles which has multiple edges sharing their source vertex and range vertex. In particular, the graph $T_{2}^{(n)}$ has noninner derivations which do not factor through any $T^{+}\left(\mathcal{C}_{w}\right)$ for any loops since there are no loops. We have the following partial converse.

PROPOSITION 8.2. Let $Q$ be a graph such that if $e$ is an edge, then either $e$ lies on a cycle or there is no other edge $f$ with $r(f)=r(e)$ and $s(f)=s(e)$. If $\theta \in \mathrm{GF}(Q)$, then if $\theta$ factors through the subalgebra $T^{+}\left(\mathcal{C}_{w}\right)$ for every cycle $w \in W$, then $\theta \in \mathrm{T}_{2} \operatorname{Inn}(Q)$.

We now remind the reader of the paper [1] where the automorphisms of $T^{+}\left(\mathcal{C}_{n}\right)$ were analyzed. The following is just a restatement of the results of [1] in language consistent with this paper, the proof can be found there.

Proposition 8.3. Let $\theta$ be an automorphism of $\mathcal{T}^{+}\left(\mathcal{C}_{n}\right)$ which is in $\mathrm{CF}\left(\mathcal{C}_{n}\right)$. Then the set MIF of those automorphisms which fix maximal ideals of $\mathcal{T}^{+}\left(\mathcal{C}_{n}\right)$ is a normal subgroup of $\mathrm{CF}\left(\mathcal{C}_{n}\right)$ and every such automorphism $\theta$ can be written as $\theta_{1} \circ \theta_{2}$ where $\theta_{1} \in \mathrm{MIF}$ and there exists a $\lambda \in \mathbb{T}$ such that for every pair of vertices $v_{i}, v_{i+1}$, the automorphism $\theta_{2}$ factors through $\mathcal{T}_{2}$ via the map $L_{i} \mapsto \lambda L_{i}$. 
In other words the diagonal 'matrices' (in this case they are scalars because there is at most one edge between a pair of vertices) associated to $\theta$ will all be the same. We are now in a position to piece all of these together for a general graph. If $e$ is an edge, then let $W_{e}$ denote the cycles in $W$ supported on $e$. Define a relation on $W$ by saying $w_{1} \sim w_{2}$ if there exists a finite sequence of edges $e_{1}, e_{2}, \ldots e_{n}$ such that $W_{e_{i}} \cap W_{e_{i+1}} \neq \emptyset$ and $w_{1} \in W_{e_{1}}, w_{2} \in W_{e_{n}}$. We say two cycles $w_{1}, w_{2} \in W$ are disjoint if they are not related via this relation.

PROPOSITION 8.4. The relation $\sim$ is an equivalence relation on $W$.

PROOF. Clearly if $w \in W$, then any edge supporting $w$ will serve to make $w \sim w$. Next, if $w_{1} \sim w_{2}$, then by reversing the sequence of edges we obtain $w_{2} \sim w_{1}$. Lastly assume that $w_{1} \sim w_{2}$ and $w_{2} \sim w_{3}$. By concatenating the two sequences of edges we obtain that $w_{1} \sim w_{3}$. Hence, $\sim$ is an equivalence relation on $W$.

Let $W_{1}, W_{2}, \ldots, W_{m}$ denote the equivalence classes of $W$ via this equivalence relationship. We now have the following proposition.

For each $1 \leq j \leq m$ and $\mu \in \mathbb{T}$ define $\Lambda_{j, \mu}$ by $\Lambda_{j, \mu}\left(P_{v}\right)=P_{v}$ for all $v \in V(Q)$, $\Lambda_{j, \mu}\left(L_{e}\right)=L_{e}$ for all edges not lying in a cycle in $W_{j}$, and $\lambda_{j, \mu}\left(L_{f}\right)=\mu L_{f}$ for all edges $f$ lying on a cycle in $W_{j}$.

Proposition 8.5. For each $1 \leq j \leq m$ and each $\mu \in \mathbb{T}$ the map $\Lambda_{\mu, j}$ extends to a continuous automorphism of $\mathcal{T}^{+}(Q)$.

Let $\operatorname{MIF}(Q)$ denote the set of those automorphism in $\mathrm{T}_{2} \operatorname{Inn}(Q)$ which, for each $w \in W$, factor through $\mathcal{T}^{+}\left(\mathcal{C}_{w}\right)$ as maximal ideal fixing automorphisms of $\mathcal{T}^{+}\left(\mathcal{C}_{w}\right)$.

PROPOSITION 8.6. The group $\operatorname{MIF}(Q)$ is a normal subgroup of $\mathrm{T}_{2} \operatorname{Inn}(Q)$ and every element of $\mathrm{T}_{2} \operatorname{Inn}(Q)$ can be written as $\theta_{1} \circ \theta_{2}$ where the automorphism $\theta_{1}$ is in $\operatorname{MIF}(Q)$ and for each equivalence class $W_{j}$ of $W$ there exists a $\mu_{j}$ such that

$$
\theta_{2}=\prod_{1 \leq j \leq m} \Lambda_{j, \mu_{j}}
$$

Proof. Let $\theta_{1}$ and $\theta_{2}$ be in $\operatorname{MIF}(Q)$ and assume $\theta \in \mathrm{T}_{2} \operatorname{Inn}(Q)$. Now let $M$ be a maximal ideal in $\mathcal{T}^{+}\left(\mathcal{C}_{w}\right)$. Then $\theta_{1} \circ \theta_{2}(M)=\theta_{1}(M)=M$ so $\theta_{1} \circ \theta_{2} \in \operatorname{MIF}(Q)$. It is clear that $\theta_{1}^{-1}(M)=M$ and, hence, $\operatorname{MIF}(Q)$ is a subgroup of $\mathrm{T}_{2} \operatorname{Inn}(Q)$. Now note that $\theta(M)$ will be a maximal ideal and, hence, $\theta \circ \theta_{1} \circ \theta^{-1} \in \operatorname{MIF}(Q)$. Hence, the subgroup is normal. The remainder of the proposition follows by applying the description of the automorphisms of $\mathcal{T}^{+}\left(\mathcal{C}_{w}\right)$ to this context.

In particular, we have the following theorem that describes the quotient group $\mathrm{T}_{2} \operatorname{Inn}(Q) / \operatorname{MIF}(Q)$.

THEOREM 8.7. For $Q$ a directed graph with equivalence classes of $W$ given by $W_{j}$ we have that

$$
\mathrm{T}_{2} \operatorname{Inn}(Q) / \operatorname{MIF}(Q) \cong \sum_{1 \leq j \leq m} \mathbb{T} .
$$




\section{Inner automorphisms}

If $\theta$ is an inner automorphism of $\mathcal{T}^{+}(Q)$, then we know that $\theta$ is continuous. We now look at how $\theta$ fits into the classification we have described above.

PROPOSITION 9.1. Let $\theta$ be an inner automorphism of $\mathcal{T}^{+}(Q)$, then $\theta \in \operatorname{MIF}(Q)$.

Proof. We first see that $\theta \in \mathrm{GF}(Q)$. This follows by noting that associated to every element of $M_{Q}$ is a unique maximal ideal. Further any inner automorphism will fix maximal ideals and hence will fix elements of $M_{Q}$.

Now since $\theta \in \operatorname{GF}(Q)$ it will factor through $\mathcal{T}^{+}\left(T_{2}^{n}\right)$ whenever $\mathcal{T}(Q)$ factors through $\operatorname{GF}(Q)$. Further, as $\theta$ is inner, it will factor through $\mathcal{T}^{+}\left(T_{2}^{(n)}\right)$ as an inner automorphism, in particular $\theta \in \mathrm{T}_{2} \operatorname{Inn}(Q)$.

Similarly we know that $\theta$ will factor as an inner automorphism through $\mathcal{T}^{+}\left(\mathcal{C}_{n}\right)$ whenever $\mathcal{T}^{+}(Q)$ does. However, the inner automorphisms of $\mathcal{T}^{+}\left(\mathcal{C}_{n}\right)$ fix the maximal ideals and, hence, $\theta \in \operatorname{MIF}(Q)$.

Lastly, recalling the conjectures of [3] and [1] concerning when an automorphism of special cases are inner, we suggest the following conjecture, one direction of which is established in the preceding section.

Conjecture. An automorphism $\theta \in \operatorname{Aut}\left(\mathcal{T}^{+}(Q)\right)$ is inner if and only if $\theta \in \operatorname{MIF}(Q)$.

\section{References}

[1] M. Alaimia, 'Automorphisms of some Banach algebras of analytic functions', Linear Algebra Appl. 298 (1999), 87-97.

[2] K. Davidson and E. Katsoulis, 'Nest representations of directed graph algebras', Proc. Lond. Math. Soc. 92(30) (2006), 762-790.

[3] K. Davidson and D. Pitts, 'The algebraic structure of non-commutative analytic Toeplitz algebras', Math. Ann. 311(2) (1998), 275-303.

[4] B. Duncan, 'Finite dimensional point derivations for graph algebras', Illinois J. Math. 52(2) (2009), 419-435.

[5] E. Katsoulis and D. Kribs, 'Isomorphisms of algebras associated with directed graphs', Math. Ann. 330 (2004), 709-728.

[6] D. Kribs and S. Power, 'Free semigroupoid algebras', J. Ramanujan Math. Soc. 19 (2004), 75-114.

[7] P. Muhly, 'A finite dimensional introduction to operator algebras', in: Operator Algebras and Applications (SAMOS, 1996) (Kluwer Academic, Dordrecht, 1997), pp. 315-354.

BENTON L. DUNCAN, Department of Mathematics, NDSU Department \# 2750, PO Box 6050, Fargo ND 58108-6050, USA

e-mail: benton.duncan@ndsu.edu 